\title{
High-temporal resolution optical observations of the gamma-ray blazar PG 1553+113
}

\author{
I. Andruchow ${ }^{1,2}$, J. A. Combi ${ }^{3}$, S. A. Cellone ${ }^{1,2}$, \\ A. J. Muñoz-Arjonilla ${ }^{4}$, G. E. Romero ${ }^{1,3}$ and J. Martí ${ }^{4}$ \\ ${ }^{1}$ Facultad de Ciencias Astronómicas y Geofísicas, Universidad Nacional de La Plata, Paseo del \\ Bosque, B1900FWA La Plata, Argentina \\ ${ }^{2}$ IALP, CONICET-UNLP, CCT La Plata, Paseo del Bosque, B1900FWA La Plata, Argentina \\ ${ }^{3}$ IAR, CONICET, CCT La Plata, C.C. No. 5 (1894) Villa Elisa, Buenos Aires, Argentina \\ ${ }^{4}$ Departamento de Física (EPS), Universidad de Jaén, Campus Las Lagunillas s/n, A3, 23071 \\ Jaén, Spain
}

\begin{abstract}
We present here the results of an observational photo-polarimetry campaign at optical wavelengths of the blazar PG $1553+113$, which was recently detected at very high energies $(>100 \mathrm{GeV})$ by the H.E.S.S and MAGIC $\gamma$-ray experiments.

Our high-temporal resolution data show significant variations in the linear polarization percentage and position angle at inter-night time-scales, while at shorter (intra-night) time-scales both parameters varied less significantly, if at all. Simultaneous differential photometry (at the $B$ and $R$ bands) shows no significant variability in the total optical flux.
\end{abstract}

Keywords. techniques: photometric - polarimetric, BL Lacertae objects: individual (PG 1553+113).

\section{Introduction}

PG 1553+113 is classified as a BL Lac object based on its featureless spectrum Miller \& Green (1983) and significant optical variability Miller et al. (1988). It has been well studied from radio to very high energy (VHE), including several simultaneous multiwavelength campaigns Osterman et al. (2006), Mankuzhiyi et al. (2009). In X-rays, it has been detected at a number of different flux levels (e.g., Donato et al. (2001), Reimer et al. (2008)). No evidence for strong or fast flux variability has been detected in this energy range. At VHE, the object was detected with HESS Aharonian et al. (2006), and latter confirmed with MAGIC observations Albert et al. (2007). Recently, PG 1553+113 was detected in the $\mathrm{GeV}$ gamma-ray regime by the Fermi Gamma-ray Space Telescope Abdo et al. (2010). Combining archival radio, optical, X-ray an VHE $\gamma$-ray data, these authors modelled its SED, showing that it can be reasonably well fit with a simple, one-zone SSC model.

\section{Observations and Results}

We observed PG 1553+113 from April 21 to April 25, 2009 with the Calar Alto Faint Object Spectrograph (CAFOS) in imaging polarimetry mode, at the CAHA $2.2 \mathrm{~m}$. telescope, Calar Alto, Spain. Exposure times were $150-240 \mathrm{~s}(B)$ and $50-120 \mathrm{~s}(R)$.

Polarimetry: Figure 2 presents the curves for the linear polarization $(P)$ and position angle $(\theta)$ against time along the five nights. According to statistical criteria of Kersteven et al. (1976), the source resulted to be variable taking the five nights together in both, $P$ 

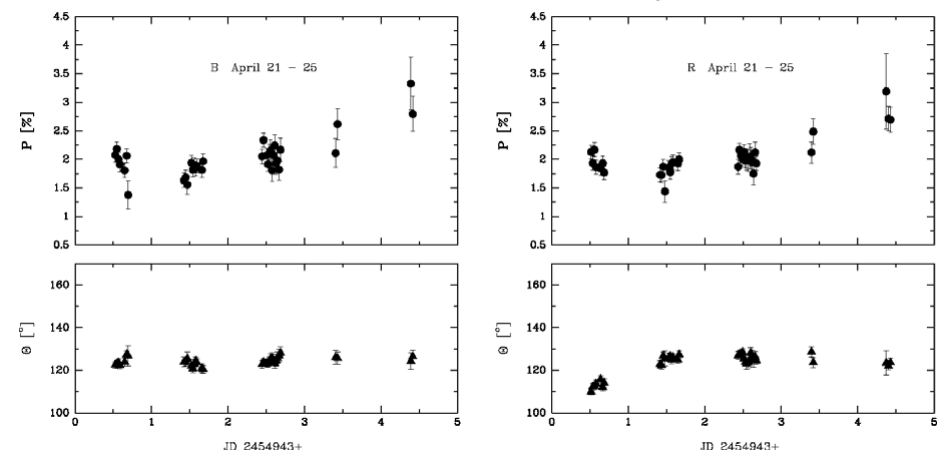

Figure 1. Polarization (top) and position angle (botton) curves for PG $1553+113$ in filters B on the left and $\mathrm{R}$ on the right

and $\theta$. The mean value for $P$ was of $2.03 \%$ and for $\theta$ was of about 127.4 in both filters. Considering the microvaribilty, PG $1553+113$ showed no important variations.

Photometry: The differential light-curves were obtained in the usual way. A statistical analysis of these light-curves reveals that PG 1553+113 showed no significant variability, neither in $B$ nor in $R$. The dispersions of the curves ranged from 0.004 to $0.013 \mathrm{mag}$, and the mean apparent magnitudes for the whole campaign were $\langle B\rangle=14.90 \pm 0.003 \mathrm{mag}$ and $\langle R\rangle=14.18 \pm 0.003 \mathrm{mag}$.

\section{Discussion}

The flux in both filters, $B$ and $R$, remained steady during the observing run here reported. For the $P$ and $\theta$ in the same period and in the same filters, the source showed small variations. Comparing our results with previous ones, ref. Osterman et al. (2006) found in their multiwavelenght campaing (April-May 2003) that PG 1553+113 was brighter in both optical bands by about $0.5 \mathrm{mag}$ showing no signs of variation. In the same period, ref. Andruchow et al. (2005) followed the source in $P$ ( $V$ filter) during two nights. $P$ and $\theta$ showed then microvariations with a most likely flickering behaviour. We note that both, $P$ and $\theta$ ere higher during 2003 and also, $P$ seems to have rotated by $\sim 20^{\circ}$ between both epochs.

The optical observations discussed in the previous paragraph bracket the VHE detections of PG $1553+113$ by HESS Aharonian et al. (2006) and MAGIC Albert et al. (2007) in 2005 and 2006 .

\section{References}

Abdo, A. A., Ackermann, M., Ajello, M., et al. 2010, ApJ, 708, 1310

Aharonian, F., Akhperjanian, A. G., Bazer-Bachi, A. R., et al. 2006, A\&A, 448, 19

Albert, J., Aliu, E., Anderhub, H., et al., 2007, ApJ, 654, L119

Andruchow, I., Romero, G. E., \& Cellone, S. A. 2005, A\& $A, 442,97$

Donato, D., Ghisellini, G., Tagliaferri, G., \& Fossati, G. 2001, A\&A A, 375, 739

Kesteven, M. J. L., Bridle, A. H., \& Brandie, G. W. 1976, AJ, 81, 919

Mankuzhiyil, N., Dorner, D., Prandini, E., et al. 2009, arXiv:0907.0740

Miller, H. R. \& Green, R. F. 1983, BAAS, 15, 957

Miller, H. R., Carini, M. T., Gaston, B. J., et al. 1988, ESA Special Publication, 281, 303

Osterman, M. A., Miller, H. R., Campbell, A. M., et al. 2006, AJ, 132, 873

Reimer, A., Costamante, L., Madejski, G., Reimer, O., \& Dorner, D. 2008, ApJ, 682, 775

Schlegel, D. J., Finkbeiner, D. P., \& Davis, M. 1998, ApJ, 500, 525 